\title{
Aspirin effect on the incidence of major adverse cardiovascular events in patients with diabetes mellitus: a systematic review and meta-analysis
}

\author{
Sonia Butalia ${ }^{1 *+}$, Alexander A Leung ${ }^{1 \dagger}$, William A Ghali ${ }^{1,2}$ and Doreen M Rabi ${ }^{1,2,3}$
}

\begin{abstract}
Background: Aspirin has been recommended for the prevention of major adverse cardiovascular events (MACE, composite of non-fatal myocardial infarction, non-fatal stroke, and cardiovascular death) in diabetic patients without previous cardiovascular disease. However, recent meta-analyses have prompted re-evaluation of this practice. The study objective was to evaluate the relative and absolute benefits and harms of aspirin for the prevention of incident MACE in patients with diabetes.

Methods: We performed a systematic review and meta-analysis on seven studies $(N=11,618)$ reporting on the use of aspirin for the primary prevention of MACE in patients with diabetes. Two reviewers conducted a systematic search of electronic databases (MEDLINE, EMBASE, the Cochrane Library, and BIOSIS) and hand searched bibliographies and clinical trial registries. Reviewers extracted data in duplicate, evaluated the quality of the trials, and calculated pooled estimates.

Results: A total of 11,618 participants were included in the analysis. The overall risk ratio (RR) for MACE was 0.91 (95\% confidence intervals, $\mathrm{Cl}, 0.82-1.00)$ with little heterogeneity among trials $\left(\mathrm{I}^{2} 0.0 \%\right)$. Secondary outcomes of interest included myocardial infarction (RR, 0.85; $95 \% \mathrm{Cl}, 0.66-1.10)$, stroke (RR, 0.84; 95\% Cl, 0.64-1.11), cardiovascular death (RR, 0.95; 95\% Cl, 0.71-1.27), and all-cause mortality (RR, 0.95; 95\% Cl, 0.85-1.06). There were higher rates of hemorrhagic and gastrointestinal events. In absolute terms, these relative risks indicate that for every 10,000 diabetic patients treated with aspirin, 109 MACE may be prevented at the expense of 19 major bleeding events (with the caveat that the relative risk for the latter is not statistically significant).

Conclusions: The studies reviewed suggest that aspirin reduces the risk of MACE in patients with diabetes without cardiovascular disease, while also causing a trend toward higher rates of bleeding and gastrointestinal complications. These findings and our absolute benefit and risk calculations suggest that those with diabetes but without cardiovascular disease lie somewhere between primary and secondary prevention patients on the spectrum of benefit and risk. This underscores the importance of considering individual risk in clinical decision making regarding aspirin in those with diabetes.
\end{abstract}

\section{Background}

Cardiovascular disease is the leading cause of mortality in patients with diabetes, accounting for an estimated $65-80 \%$ of deaths in these patients [1-3]. Although there have been substantial reductions in cardiovascularrelated morbidity and mortality in the general

\footnotetext{
*Correspondence: sbutalia@ucalgary.ca

† Contributed equally

'Department of Medicine, Faculty of Medicine, University of Calgary, Calgary, Alberta, Canada

Full list of author information is available at the end of the article
}

population over the last 40 years attributed to improved treatment of cardiovascular risk factors and disease, the same magnitude of benefit has not been observed in those with diabetes mellitus $[4,5]$. It remains unclear why certain interventions that benefit the general population may be less effective for patients with diabetes.

A landmark observational study suggested that patients with diabetes without prior myocardial infarction had a similar risk of coronary heart disease as patients with prior myocardial infarction without diabetes [6]. As a result of the ideas introduced by this 
study, significant interest emerged for the widespread implementation of interventions for lowering cardiovascular risk in patients with diabetes, such as the use of aspirin therapy. However, as others have pointed out, the widespread use of aspirin in patients with diabetes mainly reflect "extrapolations from other high risk groups... rather than on a comprehensive review of pertinent data" [7].

Several meta-analyses have explored the benefit of aspirin therapy in the primary prevention of major adverse cardiovascular events (MACE) among patients with diabetes [8-13], and concluded that there is insufficient evidence to routinely recommend aspirin therapy in patients with diabetes without known cardiovascular disease [8-10]. While these reviews provided a robust statistical summary of relative risk, some [8-10] did not report or assess aspirin effects on absolute event rates for cardiovascular and bleeding events. The latter is crucial to considering the trade-off of benefit and harm associated with aspirin. Moreover, current estimates of benefit still remain uncertain. A collaborative meta-analysis of individual patient data suggested that there was a net benefit of aspirin therapy for diabetic patients in preventing serious vascular events in the six primary prevention trials reviewed. Yet, it was noted that the results from three other primary prevention trials in diabetes did not demonstrate the same benefit [13].

In light of this uncertainty, we conducted a systematic review and meta-analysis that included data from primary prevention trials that enrolled patients from the general population as well as trials selecting for diabetic patients, as a few of these [14-16] were not previously incorporated in some of the earlier reviews $[8,10]$, and we sought to additionally quantify treatment effects in absolute terms to better-inform readers of the risk-benefit trade-off of aspirin therapy in patients with diabetes.

\section{Methods}

\section{Search strategy}

A comprehensive search was performed in MEDLINE (1950 to February 2011), PubMed, EMBASE (1980 to February 2011), and the Cochrane Library (including the Cochrane Database of Systematic Reviews, Database of Abstracts of Reviews of Effects, and Cochrane Central Register of Controlled Trials), and BIOSIS. Language restrictions were not applied, but our search was limited to human studies and randomized clinical trials using the Cochrane Collaboration filter [17]. We searched titles and abstracts with the terms "diabetes mellitus," "primary prevention," and "aspirin" as keywords (exp), and Medical Subject Headings (MeSH). The Boolean term "AND" was used to combine "diabetes mellitus" and "aspirin" as well as to combine "primary prevention" and "aspirin." The final results were then combined with the Boolean operator "OR." Bibliographies of identified studies and recent related meta-analyses were handsearched. Clinical trial registries (clinicaltrials.gov, isrctn. com, and controlled-trials.com) were also searched for ongoing and unpublished studies. Details of our search strategy are included in Additional file 1.

\section{Study selection and data abstraction}

$\mathrm{SB}$ and $\mathrm{AAL}$ independently reviewed the retrieved titles and abstracts and selected all studies reporting on the use of aspirin, the primary prevention of cardiovascular disease, and with original data reporting on a variety of cardiovascular events (see below). Full text review was then independently performed by SB and AAL for inclusion of randomized trials that compared aspirin therapy versus a cardiac-neutral comparator (e.g. placebo, vitamins), which enrolled adults ( $\geq 18$ years old), and patients with diabetes mellitus without previous historical or clinical evidence of cardiovascular disease. Studies were excluded if they were secondary publications of trials already included in the analysis, if the trial duration was twelve-months or less, or if the data (as published) could not be extracted for diabetes-specific outcomes. Disagreements in study inclusion between reviewers were resolved by consensus, and quantified.

All outcome data were extracted by SB and AAL independently, with subsequent discussion of any discrepancies. Data were collected on baseline patient characteristics, aspirin dosing and frequency, and various cardiovascular event rates (cardiovascular death, myocardial infarction, stroke, all-cause mortality, hemorrhage, gastrointestinal bleeding, and other gastrointestinal events not resulting in bleeding). Outcomes from each study were extracted in intention-to-treat categories, rather than per-protocol categories. Quality assessment was performed by extracting information on key study validity criteria [18] and a Jadad score was calculated for each study [19].

\section{Statistical analysis}

We assessed and quantified statistical heterogeneity for each outcome of interest using Cochran's Q test and the $\mathrm{I}^{2}$ statistic, respectively [20]. Analyses for the primary outcome of interest, MACE (defined as a composite of non-fatal myocardial infarction, non-fatal ischemic stroke, and cardiovascular death resulting from myocardial infarction and ischemic stroke), and all-cause mortality were performed using the Mantel-Haenszel fixed effects model. The remainder of our analyses were performed using the DerSimonian and Laird random effects model because some heterogeneity was present. We calculated risk ratios (RR) and 95\% confidence intervals (95\% CIs) for MACE, total myocardial infarction, total stroke, cardiovascular death, all-cause mortality, 
hemorrhage, gastrointestinal bleeding, and other gastrointestinal events not resulting in bleeding. We subsequently contextualized our results by calculating the absolute risk reduction (ARR, derived by calculating the difference between the combined control arm event rate and the product of the pooled relative risk and the combined control arm event rate), the number need to treat (NNT) and then compared our estimated to published data. The balance of risk and benefit was further represented by the "likelihood of being helped versus harmed" metric (LHH, a ratio of number needed to harm, as estimated by the Antithrombotic Trialists' (ATT) Collaboration [13], divided by the NNT) [21,22].

Subgroup analyses were conducted to examine the effects of aspirin dosage, allocation concealment, randomization, blinding, and whether a trial exclusively enrolled patients with diabetes on the pooled RR. We performed meta-regression analyses using maximum likelihood estimation. Assessment for publication bias was performed for the main outcome of interest with Egger's linear regression test [23,24]. Analyses were performed using Stata version 11 (StataCorp, College
Station, Texas). The study is reported according to PRISMA guidelines [18].

\section{Results}

Study selection and evaluation

Of the 4129 citations identified in our search, fifteen were identified for full-text review, and a total of seven unique trials were eligible for inclusion in this study (see Figure 1 for PRISMA flow chart). Follow-up searches failed to identify any additional trials that met inclusion criteria. Disagreement among the two reviewers regarding eligibility of studies occurred on only five occasions ( $\kappa$ score $=0.89$ ).

\section{Studies included in the systematic review}

Summary data from the seven randomized clinical trials $(\mathrm{n}=11,618)$ are presented in Table 1 [14-16,25-29]. The Physicians' Health Study (PHS) recruited male physicians, approximately $2 \%$ of whom had diabetes [29], and the Women's Health Study (WHS) enrolled women with approximately $3 \%$ of trial participants identified to have diabetes [27]. The Hypertension Optimal Treatment

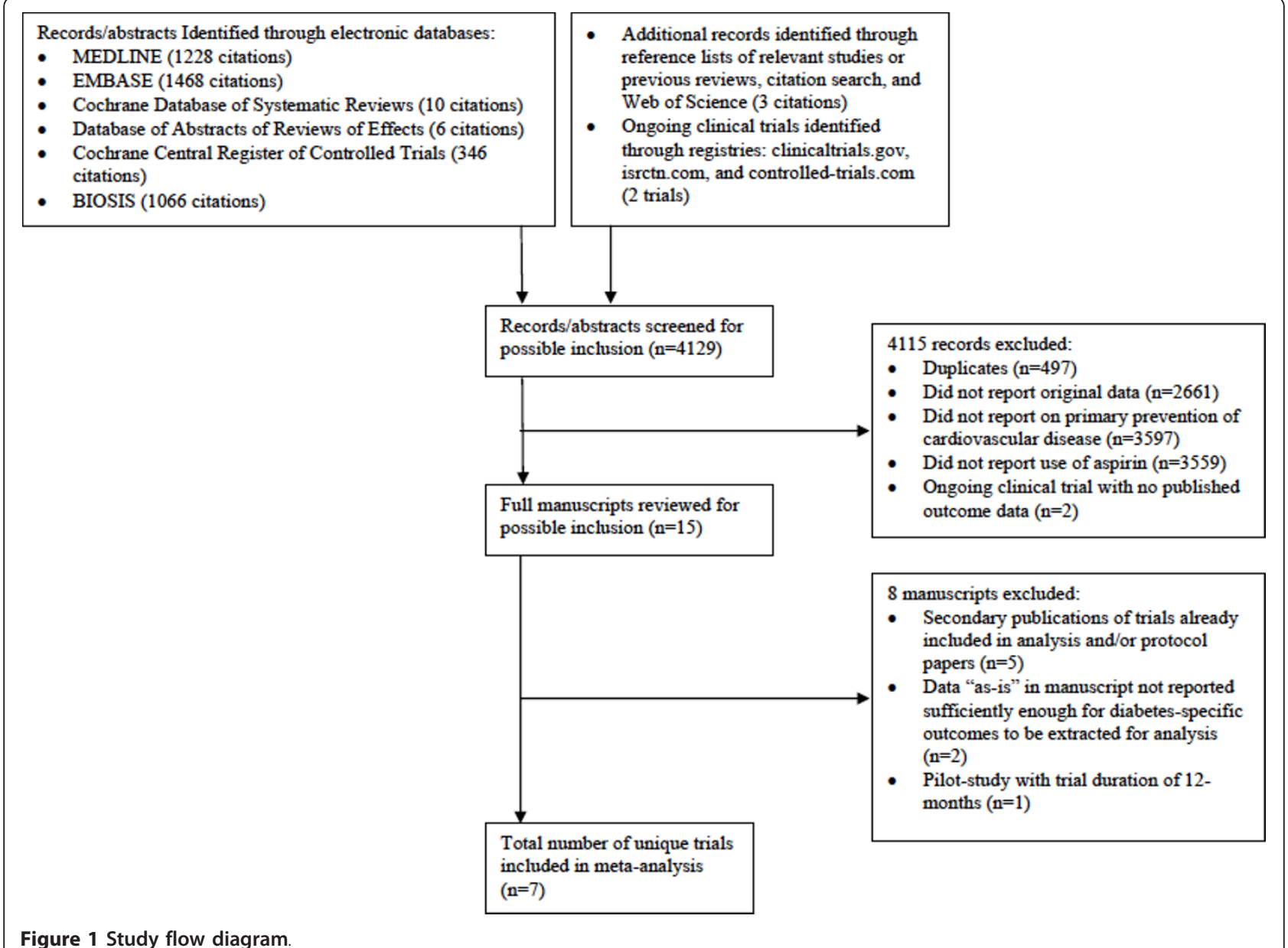

Figure 1 Study flow diagram. 


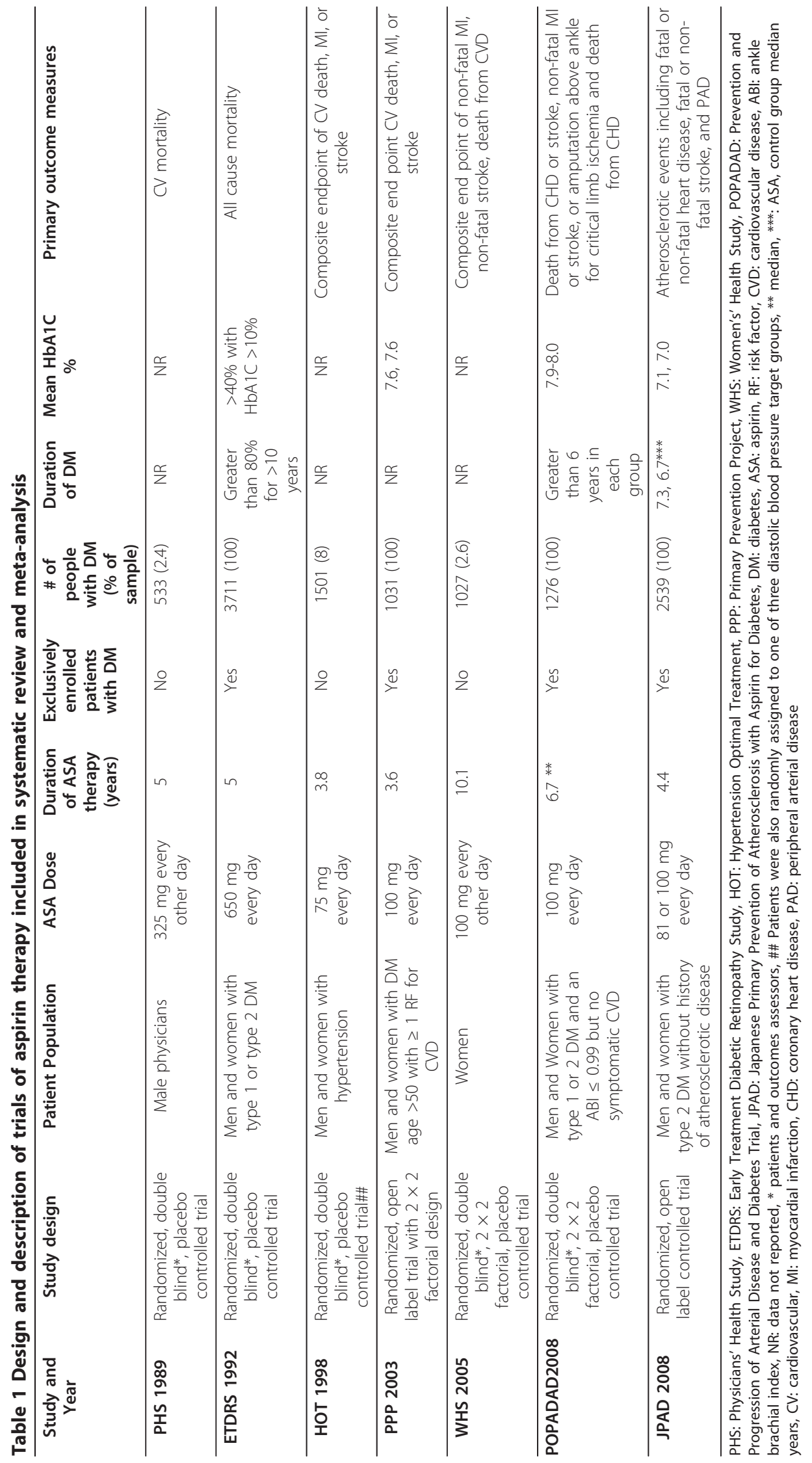


(HOT) trial studied whether the addition of aspirin to antihypertensive treatment reduced the risk of MACE compared with placebo $[14,15]$. The remaining four trials $[16,25,26,28]$ exclusively studied patients with diabetes, with two trials studying type 2 diabetes only $[25,28]$, and two trials incorporating patients with type 1 or type 2 diabetes $[16,26]$. Approximately half of the participants in the Early Treatment Diabetic Retinopathy Study (ETDRS) were reported to have a history of cardiovascular disease (i.e. self-reported history of coronary disease, heart failure, or peripheral arterial disease, as well as patients on any beta-adrenergic antagonist, antihypertensive agent, antiarrhythmic agents, digitalis, or nitrates) [16]. The remaining six trials (as reported) did not enroll any patients with overt cardiovascular disease. There was substantial variability in aspirin dosages with higher doses of $325 \mathrm{mg}$ every other day in the PHS [29] and $650 \mathrm{mg}$ daily in the ETDRS [16]. Aspirin doses of $100 \mathrm{mg}$ or less per day were used in the remaining five trials: Primary Prevention Project (PPP) [28], WHS [27], HOT [14,15], Prevention and Progression of Arterial Disease and Diabetes Trial (POPADAD) [26], and Japanese Primary Prevention of Atherosclerosis with Aspirin for Diabetes (JPAD) [25]. Trial duration ranged from 3.6 years [28] to 10.1 years [27].

A summary of study quality indicators is presented in Table 2. Randomization occurred in all seven studies, but the use of allocation concealment was clearly stated in only five of the trials [14-16,26,28,29]. All seven studies had a Jadad score of three or greater. Intention-totreat analysis was explicit in four trials $[16,25,27,28]$ and loss to follow-up was accounted for in all trials except one [28]. Treatment in three studies was randomly assigned according to a $2 \times 2$ factorial design [26-28]. The WHS [27], PPP [28], and POPADAD [26] trials also had a vitamin or anti-oxidant component. Two trials were open-labeled $[25,28]$. None of the trials appeared to have substantial baseline differences between patients allocated to aspirin therapy versus the comparator-arm.

\section{Major adverse cardiovascular events}

Six trials reported data on MACE [14-16,25-28]. A total of 612 MACE occurred among the 5565 participants with diabetes treated with aspirin compared to 668 MACE among 5520 diabetic participants in the control group (Table 3; Figure 2). Tests for statistical heterogeneity of results across the six trials revealed homogeneity $\left(\mathrm{I}^{2}=0.0 \% ; \mathrm{p}=0.945\right)$. None of the individual trials reported a significantly decreased risk of MACE among participants assigned to aspirin. However, the pooled effect estimate was very nearly significant with a relative risk (RR) of 0.91 ( $95 \% \mathrm{CI}, 0.82$ to 1.00 ).

\section{Myocardial infarction}

A total of 406 myocardial infarctions (non-fatal and fatal) was documented among 5840 participants in the aspirin group compared to 457 myocardial infarctions among the 5778 participants in the control group across the seven trials (Table 3; Figure 2) [14-16,25-29]. Heterogeneity of the trial results was moderate to high $\left(\mathrm{I}^{2}=\right.$ $53.1 \% ; \mathrm{p}=0.046)$, which may be in part due to the WHS [27] (15\% weight) and the PHS [29] (10\% weight) trials, which enrolled exclusively women and men, respectively. The pooled risk estimate of the total available data was non-significant (RR, $0.85 ; 95 \% \mathrm{CI}, 0.66$ to 1.10). After exclusion of WHS [27] and PHS [29], heterogeneity substantially decreased between trials $\left(\mathrm{I}^{2}=\right.$ $14.2 \% ; \mathrm{p}=0.324)$ with no substantial change in the overall effect estimate (RR, 0.89; 95\% CI, 0.75 to 1.06 ).

Table 2 Summary of quality indicators for studies assessing aspirin in patients with diabetes for the primary prevention of major adverse cardiovascular events

\begin{tabular}{|c|c|c|c|c|c|c|c|}
\hline $\begin{array}{l}\text { Study, } \\
\text { Year }\end{array}$ & $\begin{array}{l}\text { Allocation } \\
\text { Concealment }\end{array}$ & $\begin{array}{l}\text { Blinding of participants } \\
\text { and outcome-assessors }\end{array}$ & $\begin{array}{l}\text { Placebo - } \\
\text { controlled }\end{array}$ & $\begin{array}{l}\text { Intention to } \\
\text { Treat Analysis }\end{array}$ & $\begin{array}{l}\text { Lost to Follow } \\
\text { up Accounted }\end{array}$ & $\begin{array}{l}\text { Potential } \\
\text { Baseline } \\
\text { Difference }\end{array}$ & $\begin{array}{l}\text { JADAD Score } \\
\text { (Range 0-5) }\end{array}$ \\
\hline PHS, 1989\# & Yes & Yes & Yes & Undetermined & Yes & No & 5 \\
\hline $\begin{array}{c}\text { ETDRS, } \\
1992\end{array}$ & Yes & Yes & Yes & Yes & Yes & No & 4 \\
\hline HOT, 1998 & Yes & Yes & Yes & Undetermined & Yes & No & 4 \\
\hline PPP, 2003\# & Yes & No & $\mathrm{No}^{*}$ & Yes & Undetermined & No & 3 \\
\hline $\begin{array}{l}\text { WHS, } \\
2005 \#\end{array}$ & Undetermined & Yes & Yes & Yes & Yes & No & 4 \\
\hline $\begin{array}{l}\text { POPADAD, } \\
2008\end{array}$ & Yes & Yes & Yes $^{* *}$ & Undetermined & Yes & No & 5 \\
\hline JPAD, 2008 & No & No & No & Yes & Yes & No & 3 \\
\hline
\end{tabular}

PHS: Physicians' Health Study, ETDRS: Early Treatment Diabetic Retinopathy Study, HOT: Hypertension Optimal Treatment, PPP: Primary Prevention Project, WHS: Women's' Health Study, POPADAD: Prevention and Progression of Arterial Disease and Diabetes Trial, JPAD: Japanese Primary Prevention of Atherosclerosis with Aspirin for Diabetes, \# methods papers reviewed, * and/or comparator of vitamin E $300 \mathrm{mg}$ once daily, **and/or comparator of anti-oxidant tablet 
Table 3 Cardiovascular and mortality outcomes in studies assessing aspirin in patients with diabetes for the primary prevention of major adverse cardiovascular events

\begin{tabular}{|c|c|c|c|c|c|c|c|c|c|c|c|}
\hline $\begin{array}{l}\text { Study and } \\
\text { Year }\end{array}$ & $\begin{array}{l}\text { Total Patients } \\
\text { with Diabetes }\end{array}$ & $\begin{array}{l}\text { MACE } \\
\text { (ASA) }\end{array}$ & $\begin{array}{c}\text { MACE } \\
\text { (Control) }\end{array}$ & $\begin{array}{l}\text { Total } \\
\text { Mortality } \\
\text { (ASA) }\end{array}$ & $\begin{array}{c}\text { Total } \\
\text { Mortality } \\
\text { (Control) }\end{array}$ & $\begin{array}{c}\text { CV } \\
\text { Mortality } \\
\text { (ASA) }\end{array}$ & $\begin{array}{c}\text { CV } \\
\text { Mortality } \\
\text { (Control) }\end{array}$ & $\begin{array}{c}\text { Total } \\
\text { MI* } \\
\text { (ASA) }\end{array}$ & $\begin{array}{l}\text { Total MI* } \\
\text { (Control) }\end{array}$ & $\begin{array}{c}\text { Total } \\
\text { Stroke* } \\
\text { (ASA) }\end{array}$ & $\begin{array}{c}\text { Total } \\
\text { Stroke } \\
\text { (Control) }\end{array}$ \\
\hline PHS, 1989 & 533 & - & - & - & - & - & - & 11 & 26 & - & - \\
\hline $\begin{array}{c}\text { ETDRS, } \\
1992\end{array}$ & 3711 & 333 & 361 & 340 & 366 & 244 & 275 & 241 & 283 & 92 & 78 \\
\hline HOT, 1998 & 1501 & 47 & 54 & 40 & 36 & 23 & 26 & 11 & 18 & 20 & 22 \\
\hline PPP, 2003 & 1031 & 14 & 20 & 25 & 20 & 10 & 8 & 5 & 10 & 9 & 10 \\
\hline WHS, 2005 & 1027 & 51 & 55 & - & - & - & - & 36 & 24 & 15 & 31 \\
\hline $\begin{array}{l}\text { POPADAD, } \\
2008\end{array}$ & 1276 & 127 & 132 & 94 & 101 & 43 & 35 & 90 & 82 & 37 & 50 \\
\hline JPAD, 2008 & 2539 & 40 & 46 & 34 & 38 & 1 & 10 & 12 & 14 & 28 & 32 \\
\hline
\end{tabular}

MACE: major adverse cardiovascular events, ASA: aspirin, CV: cardiovascular, MI: myocardial infarction, *: non-fatal and fatal, " - ": not reported, PHS: Physicians' Health Study, ETDRS: Early Treatment Diabetic Retinopathy Study, HOT: Hypertension Optimal Treatment, PPP: Primary Prevention Project, WHS: Women's' Health Study, POPADAD: Prevention and Progression of Arterial Disease and Diabetes Trial, JPAD: Japanese Primary Prevention of Atherosclerosis with Aspirin for Diabetes.

\section{Ischemic stroke}

Among the 5565 participants assigned to aspirin therapy, a total (non-fatal ischemic and fatal ischemic) of 201 strokes occurred, compared to 223 strokes in the control group of 5520 participants (Table 3; Figure 2) [11-13,20-23]. Moderate amounts of heterogeneity between study results were quantified $\left(\mathrm{I}^{2}=47.4 \%\right.$; $\mathrm{p}=$ $0.091)$. Some heterogeneity may be explained by the WHS (14\% weight), a trial of women only and with the greatest relative risk reduction of strokes with aspirin [27]. The pooled RR of the results from the five trials was non-significant $(0.84 ; 95 \% \mathrm{CI}, 0.63$ to 1.11$)$. After exclusion of the WHS [27], heterogeneity diminished ( ${ }^{2}$ $=0.0 \% ; \mathrm{p}=0.468)$ and the pooled effect estimate remained non-significant.

\section{Cardiovascular death}

Death directly attributed to cardiovascular disease was found in 321 participants treated with aspirin, and 354 in the control group across five trials (Table 3; Figure 2) [14-16,25,26,28]. Only JPAD, a trial of Japanese patients, reported a statistically significant risk reduction with aspirin therapy [25]. Moderate amounts of heterogeneity were quantified in this analysis $\left(\mathrm{I}^{2}=41.1 \%\right.$; $\left.\mathrm{p}=0.148\right)$. The pooled estimate of RR of the results from the four trials was non-significant $(0.95 ; 95 \% \mathrm{CI}, 0.71$ to 1.27$)$. Although further exclusion of JPAD [25] resulted in a reduction in statistical heterogeneity $\left(\mathrm{I}^{2}=0.0 \%, \mathrm{p}=\right.$ $0.507)$, the overall effect estimate remained broadly similar (RR, 0.93; 95\% CI, 0.80 to 1.07 ).

\section{All-cause mortality}

A total of 533 deaths were recorded among 5027 participants treated with aspirin compared to 561 deaths in the control arm with 5031 participants (Table 3; Figure 2)
[14-16,25,26,28]. None of the trials reported significant reductions in risk of death associated with aspirin therapy. Heterogeneity was non-significant between trials $\left(\mathrm{I}^{2}\right.$ $=0.0 \% ; \mathrm{p}=0.838)$. The pooled effect estimate was also non-significant (RR, $0.95 ; 95 \% \mathrm{CI}, 0.85$ to 1.06 ).

\section{Drug-related side effects}

Hemorrhagic complications were uncommon. A total of 81 bleeding events occurred among 3637 participants treated with aspirin compared to 48 hemorrhagic complications among the 3644 participants treated with placebo, for a pooled RR risk of 2.50 (95\% CI, 0.77-8.10) (Table 4). 48 cases of gastrointestinal bleeding were documented among 2419 participants on aspirin compared to 36 cases among 2427 in the control group (RR 2.13, 95\% CI 0.63-7.25). Gastrointestinal events not resulting in bleeding (such as dyspepsia, non-bleeding peptic ulcers, or gastritis) occurred in 120 of 1900 participants on aspirin compared to 98 of 1915 participants in the control arm (RR 2.92 (0.17-50.23). None of these differences were statistically significant (Table 4), but statistical power was limited given the relatively low frequency of these adverse events.

\section{Absolute benefit and harm}

The ARR of MACE in the reviewed studies is 1.09\% associated with aspirin therapy with a corresponding number needed to treat (NNT) of 92 to prevent one major cardiovascular event. The estimate of the $\mathrm{LHH}$ is 6 (range of 2-8).

\section{Subgroup analysis}

Subgroup analysis was performed to explore potential sources of variability between trials. There was no statistical significant effect on the pooled RR when analyzed 


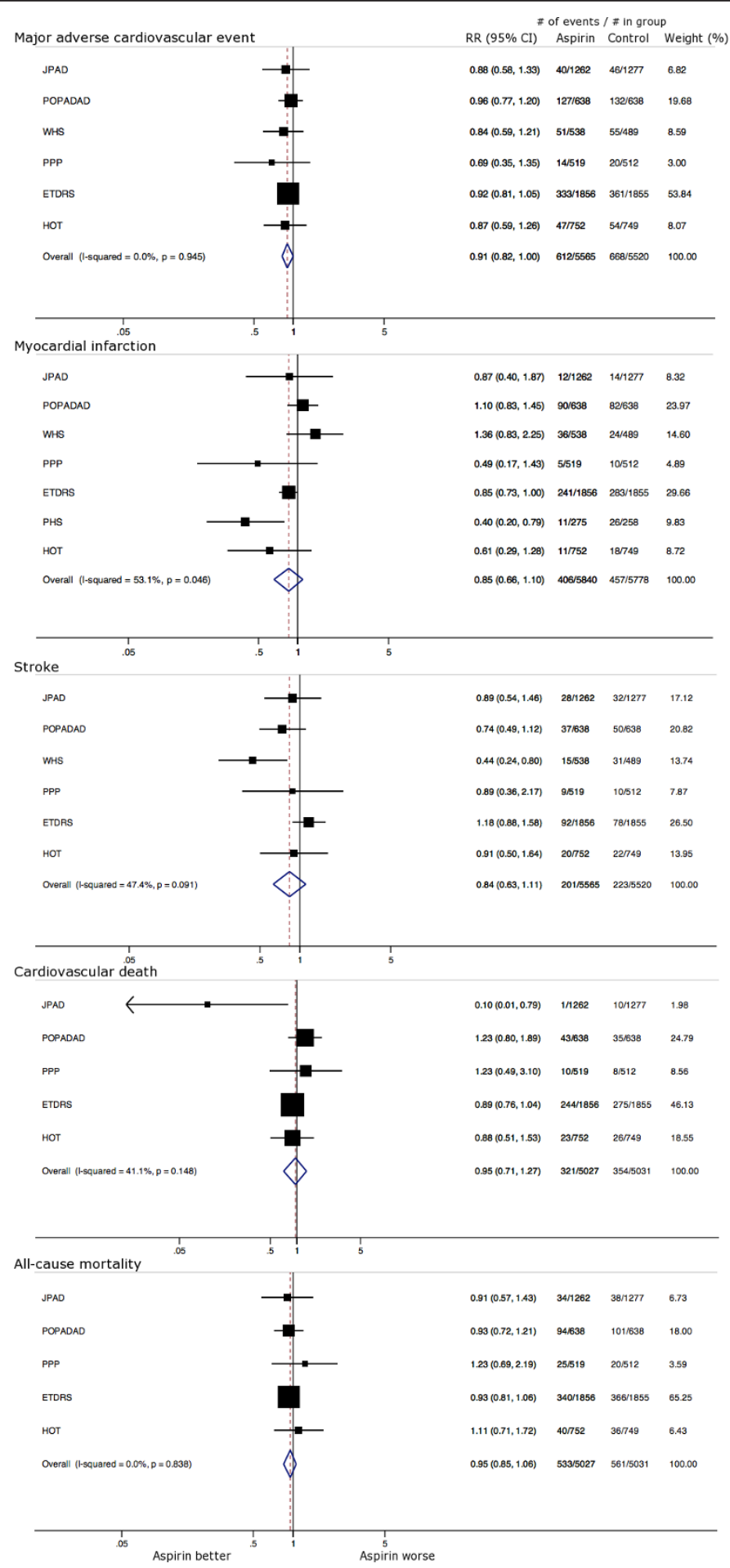

Figure 2 Effect of aspirin on the primary prevention of myocardial infarction (non-fatal and fatal), stroke (non-fatal and fatal), cardiovascular death, major adverse cardiovascular events (a composite of non-fatal myocardial infarction, non-fatal stroke, and cardiovascular death), and all-cause mortality in patients with diabetes. Relative risks (RRs) are indicated by squares with the relative weight of each trial represented by the size of each square. 95\% confidence intervals (Cls) are indicated by horizontal lines. Pooled risk estimates and corresponding $95 \% \mathrm{Cls}$ are represented by diamonds. Squares or diamonds to the left of the solid line indicate benefit with aspirin therapy. $J P A D=$ Japanese Primary Prevention of Atherosclerosis with Aspirin for Diabetes; POPADAD = Prevention Of Progression of Arterial Disease And Diabetes; WHS = Women's Health Study; PPP = Primary Prevention Project; ETDRS = Early Treatment Diabetic Retinopathy Study; HOT $=$ Hypertension Optimal Treatment; and PHS = Physicians' Health Study. 
Table 4 Risk of adverse events in studies assessing aspirin in patients with diabetes for the primary prevention of major adverse cardiovascular events

\begin{tabular}{|c|c|c|c|c|c|c|c|}
\hline Study and Year & $\begin{array}{l}\text { Total Patients } \\
\text { with Diabetes }\end{array}$ & $\begin{array}{c}\text { All } \\
\text { Bleeding } \\
\text { (ASA) }\end{array}$ & $\begin{array}{l}\text { All Bleeding } \\
\text { (Control) }\end{array}$ & $\begin{array}{l}\text { All Gl } \\
\text { Bleeding } \\
\text { (ASA) }\end{array}$ & $\begin{array}{c}\text { All GI } \\
\text { Bleeding } \\
\text { (Control) }\end{array}$ & $\begin{array}{l}\text { Non-bleeding Gl } \\
\text { symptoms (ASA) }\end{array}$ & $\begin{array}{c}\text { Non-bleeding GI } \\
\text { symptoms (Control) }\end{array}$ \\
\hline PHS, 1989 & 533 & - & - & - & - & - & - \\
\hline ETDRS, 1992 & 3711 & 37 & 37 & - & - & - & - \\
\hline HOT, 1998 & 1501 & - & - & - & - & - & - \\
\hline PPP, 2003 & 1031 & 10 & 1 & 8 & 1 & - & - \\
\hline WHS, 2005 & 1027 & - & - & - & - & - & - \\
\hline POPADAD, 2008 & 1276 & - & - & 28 & 31 & 73 & 94 \\
\hline JPAD, 2008 & 2539 & 34 & 10 & 12 & 4 & 47 & 4 \\
\hline $\begin{array}{c}\text { Number of Events/ } \\
\text { Number of Participants }\end{array}$ & - & $81 / 3637$ & $48 / 3644$ & $48 / 2419$ & $36 / 2427$ & $120 / 1900$ & $98 / 1915$ \\
\hline Pooled RR $(95 \%$ Cl) & - & \multicolumn{2}{|c|}{$2.50(0.77-8.10)$} & \multicolumn{2}{|c|}{$2.13(0.63-7.25)$} & \multicolumn{2}{|c|}{$2.92(0.17-50.23)$} \\
\hline
\end{tabular}

ASA: aspirin, Gl: gastrointestinal, PHS: Physicians' Health Study, ETDRS: Early Treatment Diabetic Retinopathy Study, HOT: Hypertension Optimal Treatment, PPP: Primary Prevention Project, WHS: Women's' Health Study, POPADAD: Prevention and Progression of Arterial Disease and Diabetes Trial, JPAD: Japanese Primary Prevention of Atherosclerosis with Aspirin for Diabetes, $R R=$ relative risk (RRs $>1$ indicate increase risk of an adverse event associated with aspirin).

according to aspirin dosage, allocation concealment, or whether a trial exclusively enrolled patients with diabetes. Randomization and blinding (of participants or outcome assessors) were also found to be non-significant covariates of heterogeneity (data not shown).

\section{Publication bias}

We assessed for publication bias by Egger's linear regression test. The $\beta$-coefficient of the bias estimate was not statistically significant $(\beta$-coefficient $=-0.72$; 95\% CI, -1.52 to $0.09 ; \mathrm{p}=0.07)$. The PHS trial was not included in the test for publication bias because MACE data were not reported [29].

\section{Discussion}

This meta-analysis of seven randomized clinical trials, combining data from 11,618 participants, indicates that aspirin therapy in patients with diabetes leads to a $9 \%$ relative reduction in the risk of major adverse cardiovascular events, a pooled risk reduction estimate that verges on statistical significance. The point estimates of aspirin effect on each of the components of the MACE composite endpoint also all favor aspirin therapy, suggesting a signal of benefit, though the analyses for each of the individual endpoints does not meet statistical significance. Drug-related side effects were relatively uncommon and not consistently reported across trials, but point estimates of effect suggest a trend toward increased risk of hemorrhage and adverse gastrointestinal events among participants treated with aspirin.

These results are not inherently surprising and while consistent with other recently published meta-analyses addressing the same clinical question [8-13], also extend findings of prior reviews. It is not surprising that several investigators have concurrently conducted systematic reviews to help answer such an important, yet unresolved question as "Is aspirin effective at preventing cardiovascular events in patients with diabetes who have not had a prior event?" While other investigators reviewing this topic have chosen to interpret statistically non-significant benefit as no evidence of benefit $[8,9]$, we view the results differently and extend the findings to provide (below) estimates of the balance of absolute risks and benefits.

It has been clearly demonstrated in a variety of patient populations that aspirin significantly decreases the risk of MACE while increasing the risk of bleeding complications [13]. Aspirin use has established benefits and associated harms and it is the relative trade-off of cardiovascular events prevented versus bleeding events caused by aspirin that needs to be carefully considered on the basis of patient characteristics and individualized estimates of cardiovascular risk [13]. Patients with established cardiovascular disease are at greatest risk for MACE and death, and thus benefit the most from aspirin therapy. In contrast, the absolute benefit of aspirin is substantially less pronounced in patients estimated to be at lower risk for the primary prevention of cardiovascular disease [13,30,31].

The pressing question is thus to consider where on the spectrum of absolute benefit and harm patients with diabetes without known existing cardiovascular disease fall? To date, the answer has been somewhat elusive, though our results shed some light on this question. The ARR of MACE in the studies that we reviewed is $1.09 \%$ associated with aspirin therapy with a corresponding NNT of 92 to prevent one major cardiovascular event. In comparison, patients with diabetes at greatest risk for MACE (i.e. risk approaching those in the general population with established cardiovascular disease) 
may have an associated NNT of 67; conversely, the NNT may be as high as 1667 in patients at lowest risk (i.e. risk identical to those in the general population without cardiovascular disease) [13]. Estimates of number needed to harm $(\mathrm{NNH})$ for major bleeding range from 526 to 3333 in patients treated with aspirin for secondary and primary prevention, respectively [13]. The balance of risk and benefit, represented by the "likelihood of being helped versus harmed" metric $(\mathrm{LHH})$, was estimated to be 6 (range of 2-8) with a more favorable risk-benefit balance with greater baseline risk. As such, for every 10,000 diabetic patients treated with aspirin, approximately 109 MACE may be prevented at the expense of 19 major bleeding events with no significant overall mortality benefit.

In this context, the fundamental question relating to patients with diabetes without cardiovascular disease is consideration of whether they are more like the general population without cardiovascular disease (i.e. "primary prevention cases"), or more in keeping (in terms of risk) with patients who have been included in secondary prevention studies. Our calculation of absolute benefit would suggest that those with diabetes but without prior cardiovascular disease lie in an intermediate position between a primary and secondary prevention on the spectrum of risk and benefit.

This invokes the controversial question of whether diabetes is a "coronary disease equivalent." There is widespread acceptance that diabetes is a major risk factor for cardiovascular disease [2,32-34], but there have been varying estimates of absolute risk [3]. In 1998, Haffner et al. found that patients with diabetes without prior myocardial infarction had a similar risk of coronary heart disease as patients with prior myocardial infarction without diabetes (i.e. affirming the "coronary disease equivalent" theory) [6]. However, at least twelve subsequent observational studies have reported conflicting results compared to the initial Finnish study [3], and a recent systematic review has presented data arguing against the notion of diabetes being a coronary-risk equivalent [3]. Although the literature uniformly agrees that patients with diabetes are at increased risk of cardiovascular events, it now suggests that the absolute risk is less than previously thought, and may explain why the benefit of some evidence-based "secondary prevention" treatments like aspirin are less marked when applied to patients with diabetes in a population-based manner.

In considering our review findings, there is a need to consider pathophysiological factors and a potential modifying effect of diabetes on aspirin efficacy. Patients with diabetes are felt to be at higher risk of atherothrombotic events because of endothelial dysfunction, impaired fibrinolysis, increased levels of circulating coagulation factors, and high platelet reactivity [35]. However, aspirin may have a dampened effect in some diabetic patients in preventing platelet aggregation [36-38]. Emerging experimental studies are now demonstrating the phenomenon of "resistance" to aspirin, which appears to have a greater prevalence among patients with diabetes [35]. Aspirin resistance has been correlated to long-term adverse outcomes with increased coronary heart disease, stroke, and peripheral vascular disease risk [35]. Whether higher doses of aspirin may translate to improved clinical outcomes in the setting of aspirin resistance, or if another antiplatelet agent is superior remains unclear.

There are currently two major ongoing trials, collectively enrolling more than 15,000 participants, evaluating the role of aspirin in patients with diabetes without prior cardiovascular events: Aspirin and Simvastatin Combination for Cardiovascular Events Prevention Trial in Diabetes (ACCEPT-D) [39] and A Study of Cardiovascular Events in Diabetes (ASCEND) [40]. In addition to exploring the role of aspirin in the prevention of cardiovascular events in patients with diabetes, these trials will help elucidate which patients with may be at greatest risk, and thus derive the most benefit from aspirin therapy.

\section{Strengths and limitations}

There are limitations to our study. Firstly, we were only able to incorporate a total of seven trials in our analysis, and quantified moderate amounts of heterogeneity among trials for the outcomes of interest. The trials of interest varied greatly when comparing patient populations, geographic locale, years of study, and design. Moreover, pooling of data was limited by inconsistent reporting of baseline characteristics and outcomes, differences in the metrics of reported values, and variability in definitions of the measured outcomes of interest (e.g. major bleeding). Statistically combining significantly heterogeneous data may be problematic. However, we were able to account for most of the heterogeneity in the secondary outcomes of interest by performing a sensitivity analysis and excluding specific trials from the secondary analysis. Exclusion of these trials did not meaningfully affect the pooled effect estimates. Further, our estimates of harm were derived from randomized studies of treatment efficacy. The decision to use only randomized trial data was made a priori and was based on the fact that rigorously conducted trials provide the most robust efficacy data. Consequently, we did not incorporate data from observational studies which may have enrolled more patients, provided longer follow-up, and been more reflective of harm associated with treatment. Lastly, the subgroup analysis and meta-regression we performed was underpowered, limited by the seven trials identified for this study. Therefore, the subgroup 
analysis is merely hypothesis-generating, and caution should be employed not to over-interpret the results.

\section{Conclusions}

Our systematic review summarizes the current state of knowledge regarding aspirin effects on risk of MACE and bleeding. While less than definitive, the body of literature indicates, somewhat predictably, that aspirin does reduce the risk of cardiovascular events while causing a trend toward higher rates of bleeding and gastrointestinal complications. The trade-off of these benefits and harms, in absolute terms, suggests that the diabetes patient without cardiovascular disease lies somewhere between primary versus secondary prevention patients on the spectrum of benefit and risk. We now await the results of ACCEPT-D [39] and ASCEND [40] to help derive more definitive estimates of absolute benefit and harm associated with aspirin, and more refined patientspecific estimates of the risk-benefit trade-offs.

\section{Additional material}

\section{Additional file 1: Detailed description of search strategy}

\section{Acknowledgements and Funding}

We would like to thank Ms. Diane Lorenzetti for providing advice in our search of the medical literature.

SB is supported by the Canadian Institutes for Health Research Fellowship award and the Alberta Innovates - Health Solutions Clinical Fellowship award. AAL is supported by the Alberta Innovates - Health Solutions Clinical Fellowship award and the Canadian Institutes for Health Research Fellowship award. DMR is supported by a Population Health Investigator award from Alberta Innovates - Health Solutions. WAG is supported by a Senior Scholar award from Alberta Innovates - Health Solutions and holds a Canadian Research Chair in Health Services Research

The funders had no role in study design, data collection and analysis, decision to publish, or preparation of the manuscript.

\section{Author details}

'Department of Medicine, Faculty of Medicine, University of Calgary, Calgary, Alberta, Canada. ${ }^{2}$ Department of Community Health Sciences, Faculty of Medicine, University of Calgary, Calgary, Alberta, Canada. ${ }^{3}$ Department of Cardiac Sciences, Faculty of Medicine, University of Calgary, Calgary, Alberta, Canada.

\section{Authors' contributions}

First authorship is shared between SB and AAL as both had equal contribution to study concept and design, search of the literature, data extraction, data analyses, and the drafting and revision of the manuscript. DMR and WAG contributed to all aspects of the study, from conception and design, to analysis, and review of the final manuscript.

\section{Competing interests}

The authors declare that they have no competing interests.

Received: 8 March 2011 Accepted: 1 April 2011 Published: 1 April 2011

\section{References}

1. Gerstein HC: Reduction of cardiovascular events and microvascular complications in diabetes with ACE inhibitor treatment: HOPE and MICRO-HOPE. Diabetes Med Res Rev 2002, 18(suppl 3):S82-S85.
2. Gress TW, Nieto FJ, Shahar E, Wofford MR, Brancati FL: Hypertension and antihypertensive therapy as risk factors for type 2 diabetes mellitus. Atherosclerosis Risk in Communities Study. N Engl J Med 2000, 342(13):905-912.

3. Bulugahapitiya U, Siyambalapitiya $S$, Sithole J, Idris I: Is diabetes a coronary risk equivalent? Systematic review and meta-analysis. Diabet Med 2009, 26(2):142-148.

4. Gu K, Cowie CC, Harris Ml: Diabetes and decline in heart disease mortality in US adults. JAMA 1999, 281(14):1291-1297.

5. Kahn R, Robertson RM, Smith R, Eddy D: The impact of prevention on reducing the burden of cardiovascular disease. Diabetes Care 2008, 31(8):1686-1696.

6. Haffner SM, Lehto S, Rönnemaa T, Pyörälä K, Laakso M: Mortality from coronary heart disease in subjects with type 2 diabetes and in nondiabetic subjects with and without prior myocardial infarction. $N$ Engl J Med 1998, 339(4):229-234.

7. Nicolucci A, De Berardis G, Sacco M, Tognoni G: AHA/ADA vs. ESC/EASD recommendations on aspirin as a primary prevention strategy in people with diabetes: how the same data generate divergent conclusions. Eur Heart J 2007, 28(16):1925-1927.

8. De Berardis G, Sacco M, Strippoli GF, et al: Aspirin for primary prevention of cardiovascular events in people with diabetes: meta-analysis of randomised controlled trials. BMJ 2009, 339:b4531.

9. Zhang C, Sun A, Zhang P, et al: Aspirin for primary prevention of cardiovascular events in patients with diabetes: A meta-analysis. Diabetes Res Clin Pract 2010, 87(2):211-218.

10. Younis $\mathrm{N}$, Williams S, Ammori B, Soran $\mathrm{H}$ : Role of aspirin in the primary prevention of cardiovascular disease in diabetes mellitus: a metaanalysis. Expert Opin Pharmacother 2010, 11(9):1459-66.

11. Calvin A, Aggarwal N, Murad $\mathrm{H}$, et al: Aspirin for the primary prevention of cardiovascular evets: A systematic review and meta-analysis comparing patients with and without diabetes. Diabetes Care 2009, 32:2300-2306.

12. Pignone $M$, Alberts $M J$, Colwell JA, et al: $A$ position statement of the American Diabetes Association, a scientific statement of the American Heart Association, and an expert consensus document of the American College of Cardiology Foundation. Diabetes Care 2010, 33(6):1392-1402.

13. Antithrombotic Trialists' (ATT) Collaboration: Aspirin in the primary and secondary prevention of vascular disease: collaborative meta-analysis of individual participant data from randomized trial. Lancet 2009, 373(9678):1849-1860.

14. Hansson L, Zanchetti A, Carruthers SG, et al: Effects of intensive bloodpressure lowering and low-dose aspirin in patients with hypertension: principal results of the Hypertension Optimal Treatment (HOT) randomised trial. Lancet 1998, 351(9118):1755-1762, HOT Study Group.

15. Zanchetti A: Aspirin and antiplatelet drugs in the prevention of cardiovascular complications of diabetes. Pharmacotherapy of diabetes: new developments: improving life and prognosis for diabetic patients New York (New York): Springer Science and Business Media; 2007, 211-218.

16. ETDRS Investigators: Aspirin effects on mortality and morbidity in patients with diabetes mellitus. Early Treatment Diabetic Retinopathy Study report 14. JAMA 1992, 268(10):1292-1300, ETDRS Investigators.

17. Higgins JPT, Green S, (editors): Cochrane Handbook for Systematic Reviews of Interventions Version 5.0.2 2009 [http://www.cochrane-handbook.org], updated September 2009. The Cochrane Collaboration.

18. Moher D, Liberati A, Tetzlaff J, Altman DG, for the PRISMA Group: Preferred reporting items for systematic reviews and meta-analyses: the PRISMA statement. Ann Intern Med 2009, 151(4):264-269.

19. Jadad AR, Moore RA, Carroll D, et al: Assessing the quality of reports of randomized clinical trials: is blinding necessary? Control Clin Trials 1996, 17(1):1-12.

20. Thompson SG, Higgins JP: How should meta-regression analyses be undertaken and interpreted? Stat Med 2002, 21(11):1559-1573.

21. Akobeng AK: Communicating the benefits and harms of treatments. Arch Dis Child 2008, 93(8):710-713.

22. Demaerschalk BM: Thrombolytic therapy for acute ischemic stroke: the likelihood of being helped versus harmed. Stroke 2007, 38(8):2215-2216.

23. Egger M, Davey Smith G, Schneider M, Minder C: Bias in meta-analysis detected by a simple, graphical test. BMJ 1997, 315(7109):629-634.

24. Hayashino $Y$, Noguchi $Y$, Fukui T: Systematic evaluation and comparison of statistical tests for publication bias. J Epidemiol 2005, 15(6):235-243. 
25. Ogawa $\mathrm{H}$, Nakayama $\mathrm{M}$, Morimoto $T$, et al: Low-dose aspirin for primary prevention of atherosclerotic events in patients with type 2 diabetes: a randomized controlled trial. JAMA 2008, 300(18):2134-2141.

26. Belch J, MacCuish A, Campbell I, et al: The prevention of progression of arterial disease and diabetes (POPADAD) trial: factorial randomised placebo controlled trial of aspirin and antioxidants in patients with diabetes and asymptomatic peripheral arterial disease. BMJ 2008, 337 a1840.

27. Ridker $\mathrm{PM}$, Cook NR, Lee IM, et al: A randomized trial of low-dose aspirin in the primary prevention of cardiovascular disease in women. $N$ Engl $J$ Med 2005, 352(13):1293-1304.

28. Sacco M, Pellegrini F, Roncaglioni MC, Avanzini F, Tognoni G, Nicolucci A, for the PPP Collaborative Group: Primary prevention of cardiovascular events with low-dose aspirin and vitamin $\mathrm{E}$ in type 2 diabetic patients: results of the Primary Prevention Project (PPP) trial. Diabetes Care 2003, 26(12):3264-3272.

29. The Steering Committee of the Physicians' Health Study Research Group: Final report on the aspirin component of the ongoing Physicians' Health Study. N Engl J Med 1989, 321(3):129-135.

30. Antithrombotic Trialists' (ATT) Collaboration: Collaborative meta-analysis of randomized trials of antiplatelet therapy for prevention of death myocardial infarction, and stroke in high risk patients. BMJ 2002, 324(7329):71-86.

31. Antiplatelet Trialists' Collaboration: Collaborative overview of randomized trials of antiplatelet therapy-l: prevention of death, myocardial infarction, and stroke by prolonged antiplatelet therapy in various categories of patients. BMJ 1994, 308(6921):81-106.

32. Gu K, Cowie CC, Harris Ml: Mortality in adults with and without diabetes in a national cohort of the U.S. population, 1971-1993. Diabetes Care 1998, 21(7):1138-1145.

33. Lee WL, Cheung AM, Cape D, Zinman B: Impact of diabetes on coronary artery disease in women and men: a meta-analysis of prospective studies. Diabetes Care 2000, 23(7):962-968.

34. Kannel WB, McGee DL: Diabetes and glucose tolerance as risk factors for cardiovascular disease: the Framingham study. Diabetes Care 1979, 2(2):120-126.

35. Angiolillo DJ, Suryadevara S: Aspirin and clopidogrel: efficacy and resistance in diabetes mellitus. Best Pract Res Clin Endocrinol Metab 2009, 23(3):375-388.

36. Colwell JA: Antiplatelet agents for the prevention of cardiovascular disease in diabetes mellitus. Am J Cardiovasc Drugs 2004, 4(2):87-106.

37. Watala C, Golanski J, Pluta J, et al: Reduced sensitivity of platelets from type 2 diabetic patients to acetylsalicylic acid (aspirin)- its relation to metabolic control. Thromb Res 2004, 113(2):101-113.

38. Watala C, Pluta J, Golanski J, et al: Increased protein glycation in diabetes mellitus is associated with decreased aspirin-mediated protein acetylation and reduced sensitivity of blood platelets to aspirin. $J \mathrm{Mol}$ Med 2005, 83(2):148-158.

39. De Berardis G, Sacco M, Evangelista V, the ACCEPT-D Study Group, et al: Aspirin and simvastatin combination for cardiovascular events prevention trial in diabetes (ACCEPT-D): design of a randomized study of the efficacy of low-dose aspirin in the prevention of cardiovascular events in subject with diabetes mellitus treated with statins. Trials 2007, 8:21-29.

40. A Study of Cardiovascular Events in Diabetes (ASCEND). [http://www.ctsu. ox.ac.uk/ascend].

doi:10.1186/1475-2840-10-25

Cite this article as: Butalia et al: Aspirin effect on the incidence of major adverse cardiovascular events in patients with diabetes mellitus: a systematic review and meta-analysis. Cardiovascular Diabetology 2011 $10: 25$.

\section{Submit your next manuscript to BioMed Central and take full advantage of:}

- Convenient online submission

- Thorough peer review

- No space constraints or color figure charges

- Immediate publication on acceptance

- Inclusion in PubMed, CAS, Scopus and Google Scholar

- Research which is freely available for redistribution

Submit your manuscript at www.biomedcentral.com/submit
Ciomed Central 